\title{
IMPLANTAÇÃO DE PLANTAS DE RECICLAGEM DE RCD: ANÁLISE FINANCEIRA, FATORES DE INFLUÊNCIA E O PAPEL DOS PRINCIPAIS AGENTES
}

\section{CDW RECYCLING PLANTS: FINANCIAL ANALYSIS, INFLUENCE FACTORS AND THE ROLE OF MAIN PLAYERS}

\author{
ANDREA PARISI KERN, Dra. | UNISINOS \\ JOANA MICHELON MORESCO, M.SC. | UNISINOS \\ EDUARDO PIZONI, M.SC. | UNISINOS \\ MARCO AURÉLIO STUMPF GONZÁLEZ, Dr. | UNISINOS
}

\begin{abstract}
RESUMO
Embora alguns países apresentam resultados positivos quanto à redução na geração de resídus de construção e demolição (RCD) e/ou aumento de reciclagem, muitos ainda apresentam altas taxas de geração e baixa taxa de reciclagem, como o Brasil. Esse artigo apresenta dois estudos de casos que envolvem implantação e operação de plantas de reciclagem: a) com venda externa de agregado reciclado (RA); e b) sem venda externa de reciclado. O objetivo do trabalho consisitu em analisar resultados financeiros e tempo e retorno de investimentos em diferentes cenários, assim como fatores de influência e papéis dos agentes. Os resultados mostram o papel essencial do poder público na garantia do fluxo de chegada do resíduo na planta e saída como agregado. Entretanto, enquanto as taxas de cobrança de disposição dos resíduos e o preço de agregado naturais são relativamente baixas, o fluxo se torna um grande desafio. Ambos os casos mostram cenários com resultado financeiro positivo nos 10 primeiros anos de operação. No primeiro, os resultados apontam vantagens na planta de $75 \mathrm{t} / \mathrm{h}$ em comparação à de 50t/t. No segundo, a demanda de agregado reciclado é um fator-chave para a otimização dos custos relacionados à implantação e operação da planta de reciclagem de RCD.
\end{abstract}

PALAVRAS-CHAVE: Resíduo de Construção e Demolição (RCD); Planta de Rciclagem de RCD; Agregado Reciclado.

\begin{abstract}
Although some countries have presented positive results from strategies to reduce construction and demolition (CDW) waste generation and/or increase recycling, many have high generation rates and low recycling rates, like Brazil. This paper presents results of two case studies, involving CDW recycling plants: a) with external sale of recycled aggregate $(R A)$; and $b$ ) without external sale of $R A$, with the objective to analyze financial results, the main influence factors and the role of different players in the viability of $C D W$ recycling. Results show the essential role that must be played by the Public Power in terms of waste flow guarantee: the arrival of the waste in the recycling plants and the consumer market for the recycled product. However, while landfill disposal and natural aggregate are available at a relatively low cost, the flow guarantee is a challenge. Both cases showed a positive financial result, considering the first years of operation. In the first, results showed advantages in the $75 \mathrm{t} / \mathrm{h}$ plant compared to the $50 \mathrm{t} / \mathrm{h}$ plant. In the second, the amount of the recycled aggregate consumed is a key factor in order to optimize the costs related to the implementation and operation of the construction waste recycle plant.
\end{abstract}

KEYWORDS: Construction and Demolition Waste (CDW); CDW Recycling Plant; Recycled Aggregate. 


\section{INTRODUÇÃO}

Os impactos ambientais dos resíduos de construção e demolição (RCD) estão relacionados ao consumo excessivo de material e, quando descartados incorretamente, à contaminação do solo e da água. Os sistemas de gerenciamento de resíduos devem se concentrar principalmente na redução da geração de resíduos e, em seguida, nas práticas de reutilização e reciclagem. Para Seadon (2010), esses sistemas devem evitar o descarte de resíduos, e somente os resíduos não recicláveis devem ser enviados para aterros controlados. (Comissão Europeia, 2008).

Grandes variações nas informações de quantidades geradas, heterogeneigade do material e a informalidade do setor de construção civil são fatores muito presentes nas questões que envolvem o gerenciamento do RCD. Essas características dificultam o estabelecimento e a supervisão de metas e estratégias fundamentais para gerenciar os resíduos gerados (Cachim; Velosa; Ferraz, 2014; Ajayi et al., 2015).

As altas taxas de RCD gerado são apresentadas na literatura, em volume ou massa. Ora são indexadas pelo número de habitantes, ora por área construída, ou ainda, por volume de construção. As diferenças entre os métodos de quantificação utilizados por diferentes acadêmicos dificultam a comparação dos resultados entre os estudos. Como exemplos, Coelho e Brito (2011) apresentam a geração de RCD em Portugal como 185,6 kg/habitante/ano. Para a região de Lisboa, Melo, Gonçalves e Martins (2011) estimaram $600 \mathrm{~kg} / \mathrm{habitante} / \mathrm{ano}$. Já Bakshan et al. (2015) estimaram 38 a $42 \mathrm{~kg} / \mathrm{m}^{2}$ de construção no Líbano, e na Espanha, Ortiz et al. (2010) sugeriram o valor de $46 \mathrm{~kg} /$ $\mathrm{m}^{2}$. Em Chennai (Índia), Ram e Kalidindi (2017) estimaram a quantidade de 1,14 milhão de toneladas/ano. No Brasil, a geração de RCD foi estimada em $84.000 .000 \mathrm{~m}^{3} / \mathrm{ano}$, segundo a Associação Brasileira de Reciclagem de Resíduos de Construção e Demolição (ABRECON), ou $150 \mathrm{~kg} / \mathrm{m}^{2}$ (Pinto, 1999) e, anos depois, $86,27 \mathrm{~kg} / \mathrm{m}^{2}$ (Oliveira, 2014).

Tanto como preconizam sistemas de gerenciamento de resíduo como a legislação vigente, a redução da geração deve ser priorizada. No entanto, esta pode ser entendida como uma tarefa complexa e árdua, por envolver diferentes atores e demandar a adoção de mudanças pelos geradores (construtores) nos processos de produção e por projetistas, em questões que envolvem racionalização nos projetos (Kern et al., 2015; Kern et al., 2018; Saez et al. 2014, Alez-al. 2014, Al-hajj; Hamani, 2011).

Cabe ao poder público estabelecer metas, criar uma estrutura de fiscalização e propor políticas de incentivos. Como bons exemplos, citam-se as metas de redução estabelecidas pela União Europeia: redução de 55\%, até 2025,
$60 \%$ até 2030 e $65 \%$ até 2035 (Conselho Europeu da União Europeia, 2019). Já quanto a estratégias de redução, pode-se mencionar a diminuição de 99 milhões de toneladas de RCD para 77 milhões de toneladas, no Japão, em dez anos (CIB, 2011) e a política de aumentar os custos de descarte de RCD como forma de induzir a reciclagem em Hong Kong (Tam e Tam, 2006).

Mais da metade do RCD gerado é classificado como resíduo Classe A pela Resolução 307 do CONAMA, composto principalmente de resíduos de cerâmica vermelha e material cimentício (Ledesma et al., 2015, Ram e Kalidindi, 2017), possibilitando a reciclagem desse material em agregado reciclado (AR).

O uso do AR tem uma grande influência na conservação de recursos, pois evita a extração de matérias-primas e fornece substitutos para materiais de construção convencionais, que requerem uma quantidade significativa de matéria-prima e energia para a produção, como areia e brita de rochas (Huang et al., 2013).

Segundo Manowong (2012) e Ajayi et al. (2015), considerando as altas taxas de geração de RCD e, ao mesmo tempo, o alto consumo de agregados naturais pela indústria da construção, pesquisas sobre o gerenciamento de $R C D$, incluindo estratégias de reciclagem são extremamente oportunas.

A indústria da construção é o setor com maior potencial de absorção de RCD, com exemplos de aplicações apresentados por vários autores. Dentre as possibilidades são incluídos: base de pavimentação e subpavimento, revestimentos primários de estradas, fabricação de argamassa para assentamento, fabricação de concreto, fabricação de pré-moldados (blocos, meio-fio, canos, entre outros) e camadas de drenagem (Ulsen, 2011).

$O$ aumento da reciclagem de RCD ocorreu principalmente por meio de ações e esforços políticos destinados a compensar a escassez de recursos naturais de alguns países (Martin e Henrichs, 2015). Essas condições, juntamente com uma redução no fornecimento de agregados naturais, criaram demandas no desenvolvimento de aplicações e tecnologias de reciclagem (Bahera et al., 2014; Cachim; Velosa; Ferraz, 2014). Por exemplo, em dez anos, o Japão aumentou a taxa de reciclagem de $58 \%$ para $92 \%$ (CIB, 2011). Bélgica, Holanda, Alemanha e Dinamarca têm uma taxa de reciclagem de 80 a $90 \%$, enquanto Israel, Espanha e Portugal têm taxas mais baixas (Katz e Baum, 2011; Mália et al., 2013). De acordo com Sabai et al. (2013), os Estados Unidos reciclam até 70\% do RCD gerado.

As plantas de reciclagem de RCD (também denominadas por usinas de reciclagem) usam tecnologias 
diferentes, que empregam desde equipamentos mais simples até mais sofisticados. A classificação das plantas de reciclagem de RCD apresentada pelo Symonds Group Ltd (1999) é dividida em três níveis: Nível 1- plantas de britagem móvel; Nível 2 - uso de sistema de triagem e peneiração; Nível 3 - plantas que empregam as mais avançadas técnicas de separação de resíduos. Segundo os autores, características como baixos preços da terra, falta de penalidades por infrações e baixos preços do agregado natural são características que levam ao uso de plantas com baixa tecnologia (níveis 1 e 2).

Como exemplo da planta de reciclagem no Nível 3, podem ser citados os agregados reciclados japoneses de alta qualidade, baseados na alta tecnologia no processo de remoção de pasta de cimento de resíduos de concreto (Tomosawa e Noguchi et al., 2005). Esses processos podem ser por trituração com triturador de rotor vertical excêntrico ou brattling da pasta cimentícia por aquecimento, seguido de sua remoção por atrito e peneiramento, conforme descrito no HRM (Healting Rubbing Method) (Ulsen, 2011). Os finos gerados são caracterizados pelo alto teor de pasta de cimento e, portanto, podem ser utilizados em aplicações mais nobres, como estabilização do solo ou matéria-prima na indústria de cimento, devido ao alto teor de cálcio (Shima et al., 2005) .

Como forma de aumentar as taxas de reciclagem de RCD, Tam e Tam (2006) apresentaram o conjunto de estratégias aplicadas por Hong Kong que envolve: as maiores taxas de descarte de aterros, a criação de uma área centralizada para a reciclagem de diferentes $R C D$, o incentivo do governo para fornecer áreas para instalações de plantas de reciclagem, produção e uso de métodos inovadores de demolição, criação de pontos de entrega voluntária de resíduos, incentivo ao uso de materiais recicláveis visando ao equilíbrio da oferta e demanda de materiais reciclados por meio de legislação.

Nunes et al. (2007) argumentam que o fluxo de recebimento de RCD é um parâmetro indispensável, assim como o mercado ativo de material reciclado.

A quantidade de RCD gerada por uma região é um parâmetro fundamental para a atividade de reciclagem (Agopyan e John, 2011), mas pode variar ao longo do tempo, devido às mudanças nas condições socioeconômicas (Coelho e Brito, 2013). Além desses parâmetros, outros impactam a viabilidade de plantas de reciclagem, como cobrar ou não taxas para receber o material (resíduo), impostos públicos e o interesse de consumo pelo AR. Todos esses parâmetros envolvem incertezas que desafiam os estudos de viabilidade.
Coelho e Brito (2011) realizaram análises de custo de ciclo de vida de uma planta de reciclagem de RCD teoricamente localizada na região metropolitana de Lisboa, Portugal, considerando 60 anos de operação e capacidade de produção de 350 t de AR por hora. Os resultados indicaram que, embora exigisse um investimento significativo, o estudo mostrou retorno financeiro em 2 anos, sugerindo ser um negócio atraente. Os principais fatores que influenciam a viabilidade econômica das plantas de reciclagem mencionados por Coelho e Brito (2013b) incluem capacidade operacional, preço de venda do agregado reciclado, taxas de descarte dos resíduos nos aterros e o volume de entrada de resíduos.

O segmento de reciclagem de resíduos de construção e demolição no Brasil ainda é muito incipiente, embora seja definida e exigida pela legislação vigente: a Resolução 307/2002 do Conselho Nacional do Meio Ambiente (Brasil, 2002) e a Política Nacional de Resíduos Sólidos - PNRS de 2010.

Segundo Miranda e Ângulo (2009), a implementação da Resolução 307/2002 fez com que o número de plantas de reciclagem de RCD nas cidades brasileiras aumentasse sensivelmente, no entanto, estagnou após 2013 (ABRECON, 2015).

Em geral, as plantas brasileiras de reciclagem de RCD têm um processo simplificado e não adotam tecnologias sofisticadas (Nível 1 e Nível 2). A maioria opera com equipamentos como carregadeira ou retroescavadeira, alimentador vibratório, transportadores de correia, britador de mandíbulas ou impacto, separador magnético permanente ou eletroímã e peneira vibratória. (Miranda, 2015). O processo começa com a classificação dos resíduos (uma ou mais etapas, separando o material indesejado) normalmente realizada quando o material chega à planta, porque o resíduo chega de maneira heterogênea, sem separação na origem. Após esta etapa, o material é triturado, peneirado e transformado em RA de diferentes granulometrias.

Além de não possuir plantas de reciclagem de RCD, uma parte significativa dos estados e municípios ainda não implementou o Plano de Gerenciamento Integrado de Resíduos da Construção, conforme exigido pela legislação. Esse plano deve estabelecer os deveres das partes envolvidas: geradores, transportadores, autoridades públicas e consumidores de AR. Também pode ser mencionada a falta de legislação que incentive o consumo de material reciclado, prejudicando e comprometendo a viabilidade de plantas particulares.

Em grande parte, as plantas de reciclagem de RCD que operam no Brasil têm capacidade nominal entre 25 e $50 \mathrm{~m}^{3} / \mathrm{h}$ e são, em primeiro lugar, do tipo privadas, em segundo lugar, públicas e em terceiro através de parceria pública/privada (ABRECON, 2015). 
No entanto, segundo Nunes et al. (2007), no início dos anos $2000,52 \%$ das plantas instaladas produziam $3.000 \mathrm{~m}^{3} /$ mês de AR e apenas $11 \%$ operavam com capacidade total. Os autores estimaram que as plantas de reciclagem de RCD no Brasil operavam com apenas $55 \%$ de sua capacidade de produção. Miranda, Angulo e Caréli (2009) indicam que a administração pública no Brasil tem dificuldades em gerenciar essa atividade, principalmente devido a mudanças de gestão ou falta de interesse. Segundo os autores, como problemas de manutenção e operação das plantas são alegadas a falta de pessoal tecnicamente preparado ou a falta de recursos para a compra de peças de reposição.

Jadovski (2005) e Nunes et al. (2007) estudaram os custos e receitas de implementação e operação de plantas de reciclagem de RCD no Brasil em relação a diferentes cenários, simulando diferentes capacidades de operação de plantas públicas e privadas. Nunes et al. (2007) concluíram que plantas públicas mostraram-se economicamente viáveis; no entanto, plantas privadas podem não ser financeiramente viáveis se obtiverem apenas receita com a venda de material reciclado. Os resultados indicam que, para serem economicamente viáveis, as plantas com capacidade de $20 \mathrm{t} / \mathrm{h}$ ou menos terão que cobrar taxas mais altas quando comparadas às plantas com capacidade de produção de $100 \mathrm{t} / \mathrm{h}$. Assim, é provável que a viabilidade financeira de plantas de reciclagem de RCD com capacidade inferior a $20 \mathrm{t} / \mathrm{h}$ seja prejudicada.

Este artigo apresenta os resultados de dois estudos de caso, envolvendo análise financeira de plantas de reciclagem de RCD, em duas cidades do Rio Grande do Sul. A primeira diz respeito à implementação de uma planta privada com a venda de agregado reciclado, enquanto a segunda é proposta como uma planta pública de CDW, adaptada de um britador existente que foi usado para produzir brita natural de basalto. No segundo estudo, o agregado reciclado produzido é para consumo do município, sem venda externa.

\section{MÉTODO DE PESQUISA}

A estratégia de pesquisa foi o estudo de caso, dividido em dois casos. Com a finalidade de contextualização os pesquisadores visitaram diferentes plantas de reciclagem de RCD que operam no Rio Grande do Sul (Passo Fundo e São Leopoldo) e em São Paulo (Campinas, Araraquara, São Carlos, Americana e São Paulo) para entender como as plantas instaladas operam e obter informações sobre equipamentos, estrutura física, logística e outros. Os preços e valores considerados nos estudos são praticados pelo mercado local, com base nas informações do fornecedor e em pesquisa de preços de mercado.

\subsection{Estudo de caso 1: Implantação de planta de RCD privada com venda externa de agregado reciclado}

A análise da implantação da planta teve como principais variáveis: (i) a capacidade de operação: $50 \mathrm{t} / \mathrm{h}$ - planta de pequeno porte (PP) e $75 \mathrm{t} / \mathrm{h}$ - planta de tamanho médio (MP); (ii) a receita de uma taxa cobrada pelo recebimento de resíduos na planta. Os custos foram classificados em três categorias: implantação, operação e manutenção. Como receita, foram consideradas a taxa de venda de RA e de recebimento dos resíduos. $O$ resultado financeiro foi analisado em diferentes cenários, considerando custos de implantação, operação e manutenção e receitas, e o retorno do custo de implementação foi estimado a partir de 10 anos de custo médio da operação.

\subsubsection{Custos de implantação}

Os custos de implementação incluem compra de terreno, construção de edifícios, equipamentos (aquisição e instalação) e licenças ambientais. $O$ custo do terreno foi estimado usando o banco de dados de uma empresa de avaliação imobiliária em Novo Hamburgo (cidade localizada na região metropolitana de Porto Alegre, Estado do Rio Grande do Sul). Para a planta de pequeno porte $(50 \mathrm{t} / \mathrm{h})$ foi considerada uma área de $12.000 \mathrm{~m}^{2}$, e para a planta de médio porte $(75 \mathrm{t} / \mathrm{h}), 16.000 \mathrm{~m}^{2}$.

Os seguintes tipos de equipamento foram considerados para a planta de pequeno porte: carregadeira de 1,7 $\mathrm{m}^{3}$, correia transportadora, alimentador vibratório, britador de mandíbulas e conduto vibratório. Para a de médio porte, carregadeira com capacidade de $2,1 \mathrm{~m}^{3}$, correia transportadora, eletroímã, alimentador vibratório, triturador e reiterador de mandíbulas e conduto vibratório. $\mathrm{O}$ custo de instalação do equipamento foi considerado como $10 \%$ do custo de aquisição.

Para as duas plantas foram arbitradas construção de mesma edificação, composta por sala de recepção, 2 banheiros, 2 vestiários, refeitório e cozinha, com área construída de $85 \mathrm{~m}^{2}$, orçada através do custo unitário básico (CUB). Também foi considerada a execução de uma cerca-viva (vegetação) no perímetro do terreno. $O$ custo das licenças ambientais (instalação e operação) foi estimado com base nos valores praticados pela Fundação Estadual de Proteção Ambiental (FEPAM).

\subsubsection{Custos de operação}

Os custos de operação incluem salários e honorários trabalhistas, e os relacionados à operação de equipamentos, despesas administrativas, custos com os rejeitos (material que chega misturado ao RCD) e impostos. 
O número de funcionários foi estabelecido com base nas visitas técnicas realizadas em outras plantas, e os salários foram determinados de acordo com o salário médio de cada operário, pesquisado no site do Sistema Nacional de Emprego (SINE), considerando encargos padrão de $71,41 \%$. Para as duas plantas o estudo considerou: um gerente técnico, um ajudante, um operador de resíduos, um operador de máquina, um operador de triturador e um vendedor. Também foram contabilizados os custos de equipamentos de proteção individual (EPI) e seus períodos de vida útil.

O custo operacional do equipamento foi estimado com base em informações dos fornecedores e tarifas do mercado de combustíveis e eletricidade. Como jornada de trabalho foi levado em conta o período de 8 horas por dia, considerando 20 dias por mês.

Os custos de água, telefone e internet foram estipulados com base nas taxas cobradas pelas empresas do mercado e considerados como despesas administrativas.

O percentual de resíduos não recicláveis em relação ao RCD que chega à planta foi estabelecido em $10 \%$, conforme informações das visitas técnicas realizadas. Os custos de transporte e disposição deste material em aterros foram buscados nas empresas de transporte de resíduos que operam na cidade.

Por fim, como impostos, foram considerados o ICMS (17\%) e o PIS / COFINS (3,5\%), considerando a AR como material de construção.

\subsubsection{Custos de manutenção}

Os custos de manutenção foram aqueles relacionados à substituição de peças de equipamentos, como garras e correias, e também os custos de manutenção preventiva (como lubrificação) e depreciação de equipamentos. Informações sobre o ciclo de vida e manutenção de equipamentos foram obtidas com fornecedores. Segundo Jadovski (2005), o custo de depreciação de todos os equipamentos utilizados na planta de reciclagem de RCD pode ser calculado considerando $10 \%$ do custo de aquisição.

\subsubsection{Receitas}

Como receita foi considerada a taxa cobrada pelo recebimento de RCD na planta e os valores recebidos pela venda de agregado reciclado. Para ambos, os valores de mercado foram considerados com base nas visitas realizadas em plantas de reciclagem.

\subsubsection{Resultados financeiros e retorno dos cus- tos de implantação}

Foram simulados quatro cenários, analisando os custos e as receitas de ambas as plantas. Para todos os cenários, $80 \%$ da capacidade operacional foi considerada no primeiro ano, $90 \%$ no segundo ano e $100 \%$ nos anos seguintes. Essa decisão foi tomada devido às dificuldades nos primeiros anos de operação em relação ao desconhecimento das novas instalações.

Foram testadas duas variáveis. A primeira, relacionada à cobrança, ou não cobrança pelo recebimento de $R C D$, e a segunda relativa à capacidade operacional da planta:

a) Cenário 1: com taxa de cobrança de recebimento do RCD, operando a $100 \%$ da capacidade;

b) Cenário 2: com taxa de cobrança de recebimento do RCD, operando a $50 \%$ da capacidade;

c) Cenário 3: sem taxa de cobrança de recebimento do RCD, operando a $100 \%$ da capacidade; e

d) Cenário 4: sem taxa de cobrança de recebimento do $\mathrm{RCD}$, operando a $50 \%$ da capacidade;

O resultado financeiro médio de 10 anos de operação das plantas foi considerado para estimar o retorno do custo de implementação (payback).

\subsubsection{Custo do agregado reciclado (US $\$ / \mathrm{m}^{3}$ )}

O custo unitário do agregado reciclado (US\$ $/ \mathrm{m}^{3}$ ) foi calculado a partir dos custos anuais de operação e manutenção, considerando a produção da planta igual à capacidade operacional, equivalente a $80 \%$ e $90 \%$ nos dois primeiros anos, respectivamente. Como a capacidade da planta é informada por $t / h$, a massa específica de CDW apresentada por Monteiro et al. (2001) de $1.200 \mathrm{~kg} / \mathrm{m}^{3}$ foi utilizada para transformar em volume $\left(\mathrm{m}^{3}\right)$, considerando 8h/dia, 20 dias/mês e 12 meses/ano.

\subsection{Estudo de Caso 2: adaptação de planta de reciclagem de $R C D$, sem venda externa de agre- gado reciclado}

O segundo estudo de caso consiste na adaptação de uma área de britagem para uma planta de reciclagem de RCD utilizando o britador existente para a produção de agregado reciclado a ser consumido por determinado município para a pavimentação de estradas na região da área rural. A área pertence à Prefeitura de uma cidade da Serra Gaúcha.

O município possui uma malha viária em áreas rurais de extrema importância e alta demanda, dada a produção agrícola maciça, sendo o maior produtor de uva moscatel no Brasil. A maioria dessas estradas possui apenas o revestimento primário (brita). Devido às condições 
climáticas e ao tráfego intenso, a recolocação de brita é frequentemente necessária nessas vias. Segundo informações coletadas na Prefeitura, durante o ano de 2017 foram produzidos $27.516 \mathrm{~m}^{3}$ de brita (agregado natural) que representa um volume de $15.831 \mathrm{~m}^{3}$ de brita destinada à pavimentação de estradas na área rural.

O triturador está localizado próximo a uma pedreira, em uma área de $35.000,00 \mathrm{~m}^{2}$. Há a construção e uma edificação utilizada para o armazenamento de máquinas e questões administrativas do local. Em termos de equipamentos, há uma carregadeira, um caminhão basculante e o britador de mandíbula fixo. No total, seis funcionários operam três operadores de máquinas, dois trabalhadores auxiliam na conferência e na remoção manual de contaminantes do material que vai para o alimentador vibratório e uma pessoa é encarregada da administração.

\subsubsection{Identificação da demanda e custo}

A primeira etapa do trabalho foi identificar as adaptações necessárias para produzir agregados reciclados de RCD, com base na observação in loco e entrevistas com os envolvidos na produção de brita. Os custos de adaptação do local foram classificados em custos de implantação (construção, equipamentos, licenças), custos de operação (contratação de mão-de-obra) e custos de manutenção e foram estimados com base nos preços de mercado, no custo unitário básico de construção e nos salários pagos pela prefeitura.

\subsubsection{Custo do agregado reciclado (US\$ $/ \mathrm{m}^{3}$ )}

O custo de produção da brita de um ano foi informado pela Prefeitura, incluindo custos de manutenção de equipamentos (troca de correia, mandíbula trituradora, peneira, troca de pneus, equipamentos de segurança e outros) e custos de operação (detonação, energia, água, combustível, mão de obra direta e trabalho indireto).

Os custos de produção do agregado reciclado foi levantado a partir do volume estimado pelo uso da brita destinado à pavimentação na área rural. Os custos de manutenção foram considerados os mesmos, bem como os custos operacionais, exceto os custos de explosão e mão de obra direta. O tempo de retorno dos custos de implantação foi estimado a partir do resultado financeiro entre a produção de agregados naturais e a produção de agregados reciclados.

\section{RESULTADOS E DISCUSSÃO}

Os resultados são apresentados em duas etapas, referentes a cada um dos estudos de caso realizados:

\subsection{Estudo de Caso 1: planta de reciclagem de RCD com venda de RA}

A Tabela 1 apresenta os custos estimados para as etapas de implantação, operação e manutenção e receita, considerando as plantas de pequeno e médio porte. A receita estimada é referente à taxa de cobrança pelo recebimento de RCD e/ou venda de agregado reciclado, com base nos preços de mercado e considerando $100 \%$ da capacidade de produção das plantas.

\begin{tabular}{|l|l|l|}
\hline CUSTOS E RECEITA & $\begin{array}{l}\text { Planta de pequeno porte } \\
(\mathbf{5 0} \mathbf{t} / \mathbf{h}) \text { US\$ }\end{array}$ & $\begin{array}{l}\text { Planta de médio porte } \\
\mathbf{( 7 5} \mathbf{t} / \mathbf{h}) \text { US\$ }\end{array}$ \\
\hline CUSTO TOTAL & $\mathbf{2 . 2 3 6 . 9 7 4 , 6 9}$ & $\mathbf{2 . 7 4 3 . 3 8 3 , 6 9}$ \\
\hline Implantação (total) & $\mathbf{6 7 6 . 9 3 9 , 9 1}$ & $\mathbf{8 5 5 . 8 3 4 , 5 4}$ \\
\hline Aquisição de equipamentos & $200.166,67$ & $243.083,33$ \\
\hline Instalaçaõ de equipamentos & $14.957,00$ & $18.750,00$ \\
\hline Terreno & $325.000,00$ & $425.000,00$ \\
\hline Construção e cerca & $133.196,43$ & $165.244,35$ \\
\hline Projetos & $3.601,81$ & $3.756,86$ \\
\hline Licenças ambientais & $6.025,00$ & $6.025,00$ \\
\hline Operação (total - custos anuais) & $\mathbf{1 . 5 0 9 . 2 7 9 , 7 0}$ & $\mathbf{8 2 . 2 2 1 , 8 2}$ \\
\hline Mão de obra & $82.221,82$ & $82.221,82$ \\
\hline Equipamentos & $27.060,46$ & $39.477,96$ \\
\hline Administração & $3.468,29$ & $3.978,29$ \\
\hline Descarte de rejeitos & $38.177,40$ & $7.266,10$ \\
\hline Impostos & custo estimado em relação à receita & \\
\hline Manutenção (total custos anuais) & $\mathbf{4 4 . 7 3 0 , 0 8}$ & $\mathbf{5 2 . 0 8 1 , 4 8}$ \\
\hline Equipamentos & $2.804,95$ & $3.182,45$ \\
\hline Depreciação & $1.668,06$ & $2.025,70$ \\
\hline
\end{tabular}




\begin{tabular}{|l|l|l|}
\hline RECEITA TOTAL & $\mathbf{7 4 8 . 1 2 5 , 0 0}$ & $\mathbf{1 . 1 2 2 . 1 8 7 , 5 0}$ \\
\hline Cobrança pelo resíduo & $360.000,00$ & $540.000,00$ \\
\hline Venda de RA & $388.125,00$ & $58.212,50$ \\
\hline
\end{tabular}

Tabela 1- Custos e receitas para instalaçãa, operação e manutenção de planta de reciclagem: pequeno e médio porte Fonte: Autores

O custo de aquisição de terra foi o mais significativo entre os custos de implantação, sendo $48 \%$ na planta pequena e $49,7 \%$ na planta média. Entre os custos operacionais, os custos de mão-de-obra foram os maiores, $54 \% \mathrm{em}$ plantas pequenas e $45 \%$ em plantas médias.

Observa-se a diferença na estimativa do potencial de receita em função da diferença de capacidade de produção das duas plantas consideradas.
A Tabela 2 mostra o resultado financeiro e o payback (retorno do custo de implantação) do cenário 1, considerando que os custos de implantação ocorrem antes da operação (ano 0) e que os custos e receitas de operação e manutenção nos dois primeiros anos apresentam uma redução de $20 \%$ e $10 \%$, respectivamente.

\begin{tabular}{|c|c|c|c|c|c|c|c|c|}
\hline \multirow{2}{*}{$\begin{array}{l}\text { Aspectos } \\
\text { Financeiros }\end{array}$} & \multicolumn{4}{|c|}{ Planta de pequeno porte (PP) US\$ } & \multicolumn{4}{|c|}{ Planta de médio porte (MP) US\$ } \\
\hline & Ano 0 & Ano 1 & Ano 2 & Ano $3-x$ & Ano 0 & Ano 1 & Ano 2 & Ano $3-x$ \\
\hline & & $80 \%$ & $90 \%$ & $100 \%$ & & $80 \%$ & $90 \%$ & $100 \%$ \\
\hline $\begin{array}{l}\text { Recebimento } \\
\text { RCD }\end{array}$ & 0,0 & $288.000,0$ & $324.000,0$ & $360.00,0$ & 0,0 & $432.000,0$ & $486.000,0$ & $540.000,0$ \\
\hline Venda RA & 0,0 & $310.500,0$ & $349.312,50$ & $388.125,0$ & 0.0 & $465.750,0$ & $523.968,8$ & $582.187,5$ \\
\hline RECEITA & 0,0 & 598.500 .0 & $673.312,5$ & $748.125,0$ & 0,0 & $897.750,0$ & $1.009 .969,0$ & $1.122 .188,0$ \\
\hline Impostos & 0,0 & $63.652,50$ & $71.609,0$ & $71.609,0$ & 0,0 & $95.478,7$ & $107.413,6$ & $119.348,4$ \\
\hline Operação & 0,0 & $150.927,8$ & $150.927,80$ & $150.927,8$ & 0,0 & $182.944,2$ & $182.944,20$ & $182.944,20$ \\
\hline Manutenção & 0,0 & $4.473,0$ & $4.473,0$ & $4.473,0$ & 0,0 & $5.208,1$ & $5.208,1$ & $5.208,1$ \\
\hline Implantação & $682.964,9$ & 0,0 & 0,0 & 0,0 & $861.859,5$ & 0,0 & 0,0 & 0,0 \\
\hline CUSTO & $682.964,9$ & $219.053,0$ & $227.010,0$ & $234.966,6$ & $861.859,5$ & $283.631,1$ & $295.565,9$ & $307.500,8$ \\
\hline $\begin{array}{l}\text { Resultado } \\
\text { Financeiro }\end{array}$ & $-682.964,9$ & $379.446,5$ & $446.302,5$ & $513.158,4$ & $-861.859,5$ & $614.118,9$ & $714.402,8$ & $814,686,7$ \\
\hline $\begin{array}{l}\text { Payback } \\
\text { (anos) }\end{array}$ & \multicolumn{4}{|c|}{1,4} & \multicolumn{4}{|c|}{1,1} \\
\hline
\end{tabular}

Tabela 2 - Cenário1: receita de taxa de recebimento do RCD na planta e operação a 100\% da caácidade Fonte: Autores

Como esperado, esse cenário apresenta um resultado financeiro positivo em todos os anos de operação, dado o otimismo relacionado às estimativas de receita, que consideram tanto o recebimento de RCD quanto a venda de agregados, bem como a produção das plantas que operam em capacidade máxima.
A Tabela 3 mostra os resultados financeiros e o payback (retorno do custo de implantação) do cenário 2, simulando a produção da planta com $50 \%$ da capacidade operacional, com receita proveniente da coleta de recebimentos de RCD.

\begin{tabular}{|l|l|l|l|l|l|l|l|l|}
\hline & \multicolumn{4}{|l}{ Planta de pequeno porte (PP) US\$ } & \multicolumn{3}{l|}{ Planta de médio porte (MP) US\$ } \\
\hline $\begin{array}{l}\text { Aspectos } \\
\text { Financeiros }\end{array}$ & Ano 0 & Ano 1 & Ano 2 & Ano 3 - $\mathbf{~}$ & Ano 0 & Ano 1 & Ano 2 & Ano 3-x \\
\hline & & $\mathbf{8 0} \%$ & $\mathbf{9 0} \%$ & $\mathbf{1 0 0} \%$ & & $\mathbf{8 0} \%$ & $\mathbf{9 0 \%}$ & $\mathbf{1 0 0 \%}$ \\
\hline $\begin{array}{l}\text { Recebimento } \\
\text { RCD }\end{array}$ & 0,0 & $144.000,0$ & $162.000,0$ & $180.000,0$ & 0,0 & $216.000,0$ & $243.000,0$ & $270.000,0$ \\
\hline Venda RA & 0,0 & $155.250,0$ & $174.656,3$ & $194.062,5$ & 0,0 & $232.875,0$ & $261.984,4$ & $291.093,8$ \\
\hline RECEITA & $\mathbf{0 , 0}$ & $\mathbf{2 9 9 . 2 5 0 , 0}$ & $\mathbf{3 3 6 . 6 5 6 , 3}$ & $\mathbf{3 7 4 . 0 6 2 , 5}$ & $\mathbf{0 , 0}$ & $\mathbf{4 4 8 . 8 7 5 , 0}$ & $\mathbf{5 0 4 . 9 8 4 , 4}$ & $\mathbf{5 6 1 . 0 9 3 , 8}$ \\
\hline Impostos & 0,0 & $31.826,2$ & $35.804,5$ & $39.782,8$ & 0,0 & $\mathbf{4 7 . 7 3 9 , 3}$ & $53.706,8$ & $59.674,2$ \\
\hline
\end{tabular}




\begin{tabular}{|c|c|c|c|c|c|c|c|c|}
\hline Operação & 0,0 & $150.928,0$ & $150.928,0$ & $150.928,0$ & 0,0 & $182.944,2$ & $182.944,2$ & $182.944,2$ \\
\hline Manutenção & 0,0 & $4.473,0$ & $4.473,0$ & $4.473,0$ & 0,0 & $5.208,1$ & $5.208,1$ & $5.208,1$ \\
\hline Implantação & 682.964 .9 & 0,0 & 0,0 & 0,00 & 861.859 .5 & 0.00 & 0,00 & 0,00 \\
\hline CUSTO & 682.964 .9 & $187.227,2$ & $191.205,5$ & $195.183,8$ & $861.859,5$ & $235.891,7$ & $371.096,5$ & $247.826,5$ \\
\hline $\begin{array}{l}\text { Resultado } \\
\text { Financeiro }\end{array}$ & -682.964 .9 & $112.022,8$ & $145.450,7$ & $178.878,7$ & $-861.859,5$ & $212.983,3$ & $133.887,9$ & $313.267,2$ \\
\hline $\begin{array}{l}\text { Payback } \\
\text { (anos) }\end{array}$ & \multicolumn{4}{|c|}{4.0} & \multicolumn{4}{|c|}{3.0} \\
\hline
\end{tabular}

Tabela 3 - Cenário 3: receita de taxa de recebimento do RCD na planta e operação a 50\% da caácidade Fonte: Autores

Mesmo com a capacidade de produção reduzida pela metade, a simulação comparativa de receitas e custos apresenta resultados financeiros positivos desde o primeiro ano de operação, uma vez que a receita estimada é superior às despesas.
O terceiro cenário simula os resultados financeiros e o payback (retorno do custo de implantação), em relação a $100 \%$ da capacidade operacional, sem receita proveniente da cobrança do recebimento de RCD (Tabela 4).

\begin{tabular}{|c|c|c|c|c|c|c|c|c|}
\hline \multirow{2}{*}{$\begin{array}{l}\text { Aspectos } \\
\text { Financeiros }\end{array}$} & \multicolumn{4}{|c|}{ Planta de pequeno porte (PP) US\$ } & \multicolumn{4}{|c|}{ Planta de médio porte (MP) US\$ } \\
\hline & Ano 0 & Ano 1 & Ano 2 & Ano $3-x$ & Ano 0 & Ano 1 & Ano 2 & Ano $3-x$ \\
\hline & & $80 \%$ & $90 \%$ & $100 \%$ & & $80 \%$ & $90 \%$ & $100 \%$ \\
\hline $\begin{array}{l}\text { Recebimento } \\
\text { RCD }\end{array}$ & 0,0 & 0,0 & 0,00 & 0,0 & 0,0 & 0,0 & 0,0 & 0,0 \\
\hline Venda RA & 0,0 & $310.500,0$ & $349.312,5$ & $388.125,0$ & 0,0 & $465.750,0$ & $523.968,8$ & $582.187,5$ \\
\hline RECEITA & 0,0 & $310.500,0$ & $349.312,5$ & $388.125,0$ & 0,0 & $465.750,0$ & $523.968,8$ & $582.187,5$ \\
\hline Impostos & 0,0 & $63.652,5$ & $71.609,0$ & $150.928,0$ & 0,0 & $95.478,7$ & $107.413,6$ & $119.348,4$ \\
\hline Operação & 0,0 & $150.928,0$ & $150.928,0$ & $150.928,0$ & 0,0 & $182.944,2$ & $182.944,2$ & $182.944,2$ \\
\hline Manutenção & 0,0 & $4.473,0$ & $4.473,0$ & $4.473,0$ & 0,0 & $5.208,1$ & $5.208,1$ & $5.208,1$ \\
\hline Implantação & $682,964,9$ & 0,00 & 0,00 & 0,00 & $861.859,5$ & & 0,0 & 0,0 \\
\hline CUSTO & $682,964,9$ & $219.053,5$ & $227.010,0$ & $234.966,6$ & $861.859,5$ & $283.631,1$ & $295.565,9$ & $307.500,8$ \\
\hline $\begin{array}{l}\text { Resultado } \\
\text { Financeiro }\end{array}$ & $-682,964,9$ & $91.446,5$ & $122.302,5$ & $153.158,4$ & $-861.859,5$ & $182.118,9$ & $228.402,8$ & $274.686,7$ \\
\hline $\begin{array}{l}\text { Payback } \\
\text { (anos) }\end{array}$ & \multicolumn{4}{|c|}{4,7} & \multicolumn{4}{|c|}{3,3} \\
\hline
\end{tabular}

Tabela 4 - Cenário 3: sem receita de taxa de recebimento do RCD na planta e operação a 100\% da capacidade Fonte: Autores

Como esperado, a falta de receita com o recebimento de RCD diminui os resultados financeiros do negócio, embora seja positivo desde o primeiro ano de operação, pois a receita estimada com a venda de RCD é superior aos custos tributários, operacionais e de manutenção.
A Tabela 5 mostra os resultados financeiros e o payback (retorno do custo de implantação) do quarto cenário, que considera as plantas operando com $50 \%$ da capacidade de produção, sem receita proveniente do recebimento de CDW.

\begin{tabular}{|l|l|l|l|l|l|l|l|l|}
\hline & \multicolumn{4}{|l}{ Planta de pequeno porte (PP) US\$ } & \multicolumn{3}{l|}{ Planta de médio porte (MP) US\$ } \\
\hline $\begin{array}{l}\text { Aspectos } \\
\text { Financeiros }\end{array}$ & Ano 0 & Ano 1 & Ano 2 & Ano 3 - x & Ano 0 & Ano 1 & Ano 2 & Ano 3 - x \\
\hline & & $\mathbf{8 0} \%$ & $\mathbf{9 0} \%$ & $\mathbf{1 0 0} \%$ & & $\mathbf{8 0} \%$ & $\mathbf{9 0 \%}$ & $\mathbf{1 0 0 \%}$ \\
\hline $\begin{array}{l}\text { Recebimento } \\
\text { RCD }\end{array}$ & 0,0 & 0,0 & 0,0 & 0,0 & 0,0 & 0,0 & 0,0 & 0,0 \\
\hline Venda RA & 0,0 & $155.250,0$ & $174.656,3$ & $194.062,5$ & 0,0 & $232.875,0$ & $261.984,4$ & $291.093,8$ \\
\hline RECEITA & $\mathbf{0 , 0}$ & $\mathbf{1 5 5 . 2 5 0 , 0}$ & $\mathbf{1 7 4 . 6 5 6 , 3}$ & $\mathbf{1 9 4 . 0 6 2 , 5}$ & $\mathbf{0 , 0}$ & $\mathbf{2 3 2 . 8 7 5 , 0}$ & $\mathbf{2 6 1 . 9 8 4 , 4}$ & $\mathbf{2 9 1 . 0 9 3 , 8}$ \\
\hline
\end{tabular}




\begin{tabular}{|c|c|c|c|c|c|c|c|c|}
\hline Impostos & 0,0 & $16.511,3$ & $18.575,2$ & $20.639,2$ & 0,0 & $24.767,0$ & $27.862,9$ & $30.958,8$ \\
\hline Operação & 0,0 & $150.928,0$ & $150.928,0$ & $150.928,0$ & 0,0 & $182.944,2$ & $182.944,2$ & $182.944,2$ \\
\hline Manutenção & 0,0 & $4.473,0$ & $4.473,0$ & $4.473,0$ & 0,0 & $5.208,1$ & $5.208,1$ & $5.208,1$ \\
\hline Implantação & $682,964,9$ & 0,0 & 0,0 & 0,0 & $861.859,5$ & 0,0 & 0,0 & 0,0 \\
\hline CUSTO & 682.964 .9 & $171.912,3$ & $173.976,3$ & $176.040,2$ & $861.859,5$ & $212.919,4$ & $216.015,2$ & $219.111,1$ \\
\hline $\begin{array}{l}\text { Resultado } \\
\text { Financeiro }\end{array}$ & $-682.964,9$ & $-16.662,3$ & 679,99 & $18.022,32$ & - 861.859,5 & $19.955,64$ & $45.969,14$ & $71.982,6$ \\
\hline $\begin{array}{l}\text { Payback } \\
\text { (anos) }\end{array}$ & \multicolumn{4}{|c|}{4,7} & \multicolumn{4}{|c|}{3,3} \\
\hline
\end{tabular}

Tabela 5 - Cenário 4: sem receita de taxa de recebimento do RCD na planta e operação a 50\% da caácidade Fonte: Autores

A operação com $50 \%$ da capacidade de produção sem receita proveniente da taxa de recebimento de CDW indicou resultado negativo no primeiro ano na planta de pequeno porte, com redução significativa nos resultados nos anos seguintes em comparação com os outros cenários. A planta de médio porte apresentou resultados positivos nos primeiros anos, também com a diminuição dos valores, em comparação com os diferentes cenários
A Figura 1 apresenta os resultados financeiros dos quatro cenários, considerando os primeiros 5 anos. São mostrados os custos de implementação, os resultados financeiros e as pequenas e médias empresas de reciclagem de $R C D$ de retorno, denominadas $P P$ e $M P$, respectivamente.

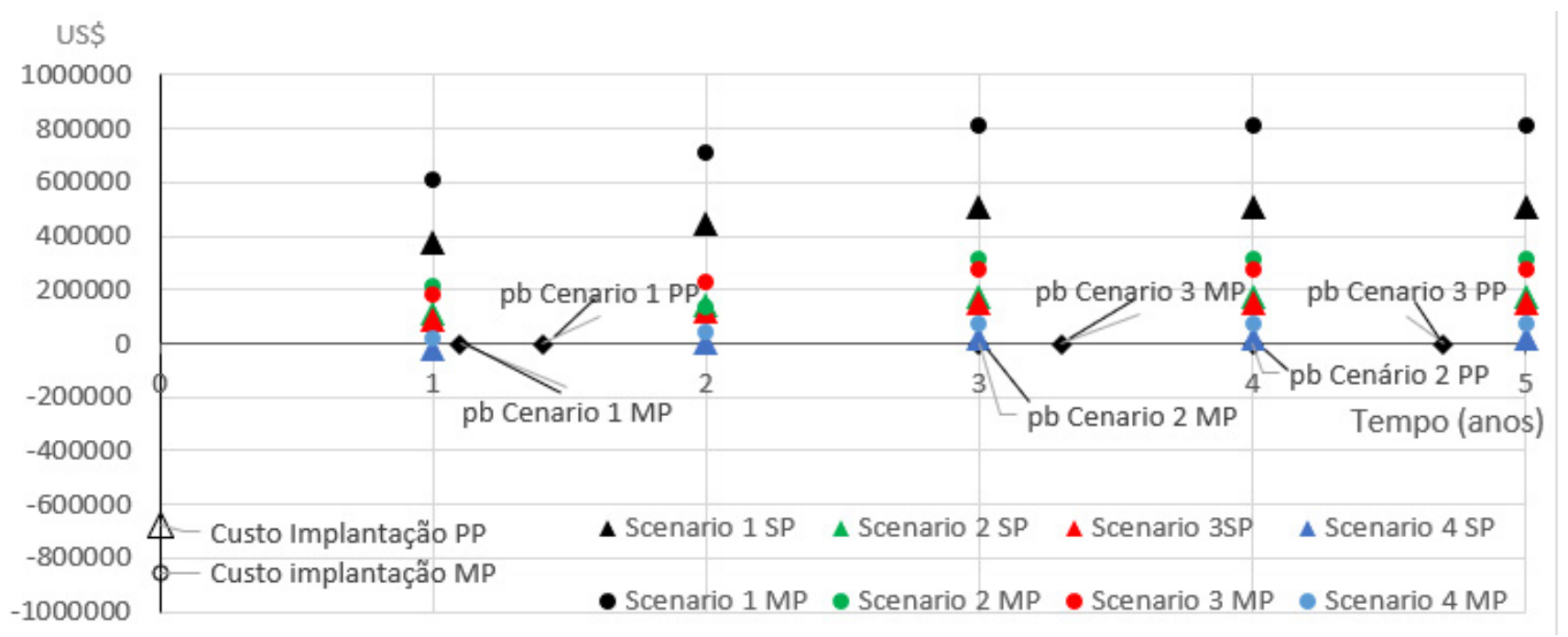

Figura 1 - Custo de implantação, resultado financeiro (5 primeiros anos) e tempo de payback ( $p b)$ Fonte: Autores

A partir da Figura 1, é possível visualizar os diferentes resultados dos cenários simulados: plantas de médio porte apresentam maior custo de implementação; no entanto, melhores resultados financeiros durante a operação em todos os cenários.

Observa-se que existe uma diferença relativamente pequena no retorno entre o Cenário 2 e 3 para o mesmo tamanho de planta, e uma diferença significativa no retorno estimado para o Cenário 4, especialmente para a pequena planta ( 53,2 anos), o que não é mostrado na figura.

\subsection{Estudo de Caso 2: Adaptação de planta de reciclagem de $R C D$, sem venda de $R A$}

As seguintes demandas foram identificadas como necessárias para adaptar o triturador existente a uma planta de reciclagem de RCD:

a) contratar dois funcionários: um para inspeção, aceitação e quantificação do RCD e das caçambas, e outro para ser responsável pelo armazenamento e classificação primária dos resíduos;

b) treinamento de funcionários em termos de controle de qualidade, operação e emergência; 
c) aquisição de eletroímã para realizar a triagem magnética após trituração do material;

d) construção de local para a recepção dos resíduos próximo à área de acesso; $\mathrm{e}$

e) construção do local de triagem e armazenamento temporário de materiais não recicláveis.

\subsubsection{Custos estimados para a adaptação à planta de reciclagem de $R C D$}

De acordo com dados da Prefeitura, o custo mensal da mão-de-obra para a contratação dos dois funcionários, trabalhando 8 h por dia (44h semanais), é de US $\$ 829,22 /$ mês, resultando em US $\$ 11.678,18 /$ ano.

A compra e instalação do eletroímã foram estimadas em US $\$ 7.500,00$, incluindo a estrutura necessária para suportá-lo, de acordo com pesquisa realizada com os fabricantes deste equipamento.

A área a ser construída foi arbitrada a partir da capacidade de produção da futura planta de reciclagem de RCD, considerando o período de armazenamento de uma semana.
Os custos de construção foram calculados através do Custo Unitário Básico (CUB) US\$12.893,93. O custo das licenças ambientais (instalação e operação) foi estimado com base nos valores praticados pela Fundação Estadual de Proteção Ambiental (FEPAM), em US\$ 6.025,00.

O custo mais alto está relacionado à construção que é considerada como custo de implementação, juntamente com a aquisição e instalação do equipamento eletroímã e os custos ambientais, resultando em US\$26.464,38. Os custos relacionados à contratação de funcionários foram considerados como custo operacional.

\subsubsection{Custo de produção de brita e Agregado Reciclado}

A Tabela 6 apresenta os custos para a produção de brita e de agregado reciclado, considerando o volume alocado para as áreas rurais, designadas para pavimentação de estradas. Também apresenta o tempo de retorno do custo de implantação (adaptação) da planta de reciclagem de RCD e o resultado financeiro anual, sendo, no primeiro ano a produção de $80 \%$ da quantidade de agregado reciclado, no segundo, $90 \%$ e nos demais $100 \%$.

\begin{tabular}{|c|c|c|c|c|}
\hline & Brita & Brita e Agregado Re & & \\
\hline & $\left(27.516 \mathrm{~m}^{3} / \mathrm{ano}\right)$ & $\begin{array}{l}\text { Ano } 1 \\
\left(14.851,20 \mathrm{~m}^{3} / \text { ano e }\right. \\
\left.12.664,80 \mathrm{~m}^{3} / \text { ano }\right)\end{array}$ & $\begin{array}{l}\text { Ano } 2 \\
\left(13.268,10 \mathrm{~m}^{3} / \mathrm{ano} \text { e }\right. \\
\left.14.247,90 \mathrm{~m}^{3} / \mathrm{ano}\right)\end{array}$ & $\begin{array}{l}\text { Anos seguintes } \\
\left(11.685,0 \mathrm{~m}^{3} / \text { ano e }\right. \\
\left.15.831,0 \mathrm{~m}^{3} / \text { year }\right)\end{array}$ \\
\hline Manutenção US\$ & $23.496,25$ & $23.496,25$ & $23.496,25$ & $23.496,25$ \\
\hline Belt & $23.496,25$ & $23.496,25$ & $23.496,25$ & $23.96,25$ \\
\hline Fixed Jaw & $7.400,00$ & $7.400,00$ & $7.400,00$ & $7.400,00$ \\
\hline Sieve & $1.155,00$ & $1.155,00$ & $1.155,00$ & $1.155,00$ \\
\hline Tires & $2.775,00$ & $2.775,00$ & $2.775,00$ & $2.775,00$ \\
\hline Others & $9.895,00$ & $9.895,00$ & $9.895,00$ & $9.895,00$ \\
\hline Operação US\$ & $304.988,81$ & $292.148,38$ & $289.271,69$ & $286.395,05$ \\
\hline Detonação & $50.000,00$ & $26.986,48$ & $24.109,79$ & $21.233,10$ \\
\hline Óleo diesel & $13.800,00$ & $13.800,00$ & $13.800,00$ & $13.800,00$ \\
\hline Energia elétrica & $27.157,93$ & $27.157,93$ & $27.157,93$ & 27.157,93 \\
\hline Água & 606,72 & 606,72 & 606,72 & 606,72 \\
\hline Mão de obra (direta) & $193.892,04$ & $193.892,04$ & $193.892,04$ & $193.892,04$ \\
\hline Mão de obra (indireta) & $9.895,00$ & $9.895,00$ & $9.895,00$ & $9.895,00$ \\
\hline Total/ano & $328.485,06$ & $315.644,75$ & $312.768,00$ & $309.891,30$ \\
\hline Resultado Financeiro & $-26.468,88$ & $12.837,81$ & $15.717,06$ & $18.593,76$ \\
\hline Total US\$/m $\mathbf{m}^{3}$ & 11,94 & 11,47 & 11,37 & 11,26 \\
\hline
\end{tabular}

Tabela 6 - Custo de manutenção e operação (produção de brita e agregado reciclado)

Fonte: Autores

A Figura 2 mostra o resultado anual da adaptação do triturador a uma planta de reciclagem de CDW, a média de dez anos e o tempo de retorno do custo de implementação. 


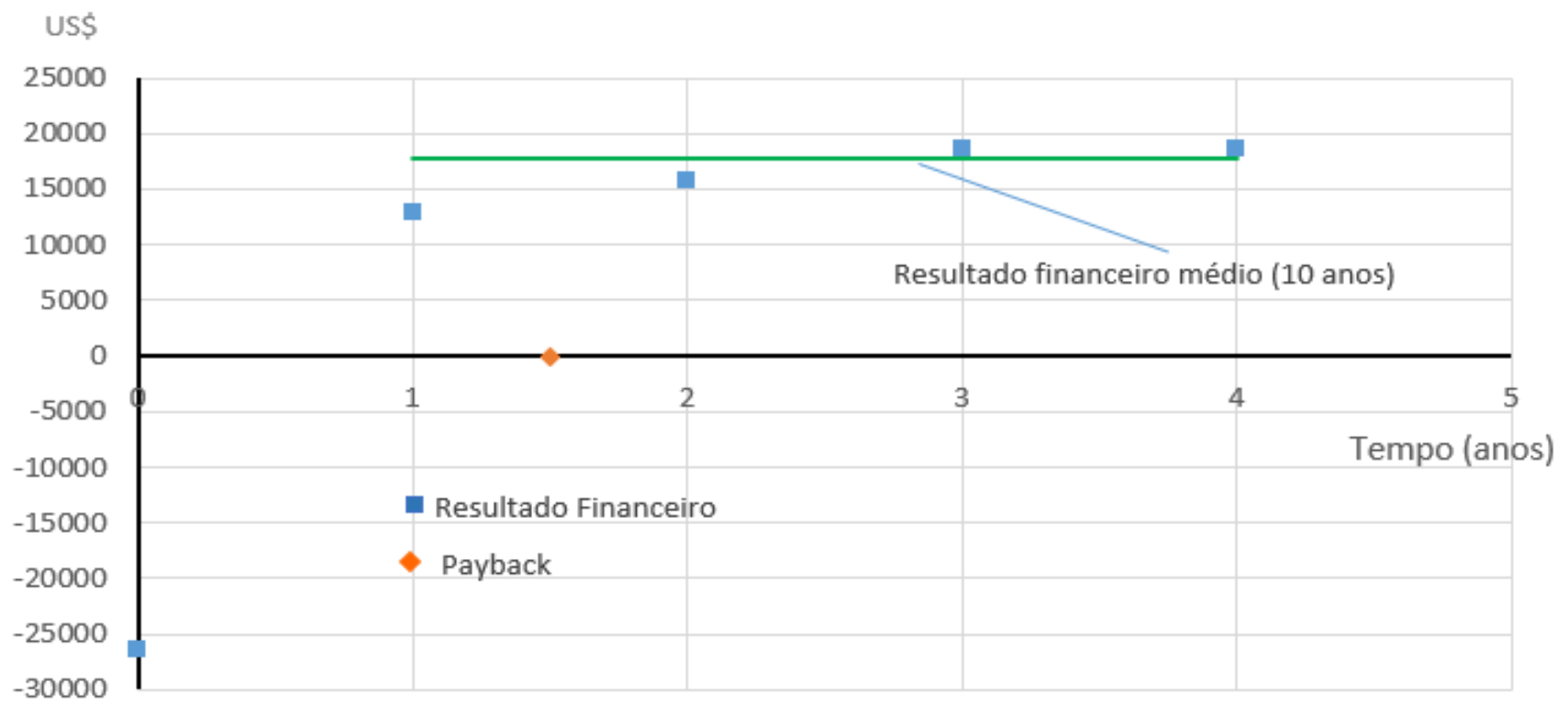

Figura 2 - Resultado finnceiro, custo de implantação e payback (pb) Fonte: Autores

De acordo com as informações disponíveis nos documentos da Prefeitura, verificou-se que aproximadamente $103.155,59 \mathrm{~m}^{2}$ de novas construções foram aprovadas no ano de 2017. De acordo com a taxa de geração de $150 \mathrm{~kg}$ $/ \mathrm{m}^{2}$ proposta por Pinto (1999), a potencial geração anual de RCD é estimado em $15.473 .488,50 \mathrm{~kg}$.

Para estimar a geração de resíduos recicláveis (agregados), foram utilizados os resultados apresentados por Ângulo (2005), que sugere a fração de $91 \%$ da massa de CDW. Assim, a geração estimada de RCD útil para a planta de reciclagem é de $14.081 .784,54 \mathrm{~kg} /$ ano.

A partir da massa específica de RCD de $1.200 \mathrm{~kg} / \mathrm{m}^{3}$ (Monteiro et al., 2001), a geração anual de resíduos recicláveis é estimada em 11.734,82 $\mathrm{m}^{3}$. Esse volume é inferior à demanda de agregado utilizado na pavimentação de estradas rurais no ano de 2017, informado como $15.831 \mathrm{~m}^{3}$. Assim, o Município, por meio de parcerias ou consórcios, deve coletar resíduos de construção e demolição gerados em outros municípios.

\section{CONCLUSÂO}

Os resultados dos diferentes cenários aplicados à implantação de uma planta de reciclagem de RCD com a venda externa de agregado reciclado mostram vantagens financeiras na planta de médio porte $(75 \mathrm{t} / \mathrm{h})$ em comparação com a planta de pequeno porte $(50 t / h)$ nos quatro cenários simulados.

O cenário que considera a receita no recebimento de $\mathrm{RCD}$, operando a $50 \%$ da capacidade de produção (Cenário 2) e a simulação que não considera receita de recebimento de RCD, operando a $100 \%$ da capacidade de produção (Cenário 3) mostram resultados financeiros e payback (retorno do investimento) relativamente similares. Isso indica que a não cobrança de taxa de recebimento de RCD pode ser relativamente compensada com a venda de uma quantidade mais significativa de agregado.

Portanto, o desafio para tornar a implantação de palntas de reciclagem de $R C D$ viáveis do ponto de vista financeiro passa pela garantia de fluxo: chegada de RCD na planta e ao mesmo tempo, demanda de consumo de agregado reciclado (saída). Não cobrar taxa de recebimento do RCD pode ser entendido como um incentivo aos geradores e transportadores, garantindo a chegada do resíduo à planta. Para o consumo de RA, entende-se que estratégias adotadas por outros países, em legislação que obriga o uso em certa quantidade e em certos tipos de obra, devam ser adotadas, e aí o poder público passa a ter um papel importante a ser desempenhado.

Destaca-se o longo tempo de retorno do investimento de implantação (payback) do Cenário 4, que não supõe cobrança pelo recebimento de $R C D$, além de operar com $50 \%$ da capacidade de produção. Trata-se do único cenário que apresenta resultado financeiro negativo no primeiro ano. Porém, com base nas pesquisas realizadas pela $A B R E C O N$, pode ser considerado o mais próximo da realidade das plantas de reciclagem de RCD no país, levando em consideração os diagnósticos realizados e a dificuldade de muitos municípios de atenderem à legislação vigente, no sentido de fiscalização.

Os resultados da adaptação do britador existente para uma planta de reciclagem de RCD para produzir agregado reciclado para consumo de uma prefeitura se mostraram 
positivos ao negócio, com retorno financeiro relativamente curto $(1,5$ anos). Isso mostra que a estrutura pré-existente (local, equipamento e mão-de-obra) adaptada para receber e reciclar RCD é vantajosa no sentido de desonerar custos de implentação da planta, além do desenho do negócio não depender de consumo externo.

O principal fator de influência observado nos dois estudos está relacionado ao fluxo de resíduos (entra como RCD e sai como agregado reciclado), envolvendo três principais agentes: geradores, recicladores e consumidores. O Poder Público, por sua vez, desempenha um papel essencial em termos de garantia do fluxo de resíduos, exigindo estratégias para garantir que geradores e transportadores enviem os resíduos gerados para as plantas de reciclagem e, ao mesmo tempo, políticas para criar um mercado consumidor para o produto reciclado, via incentivos ou obrigações. Embora o agregado natural esteja disponível a um custo relativamente baixo, a criação do mercado consumidor pode ser entendida como um desafio significativo.

Os resultados mostram que a separação dos resíduos pelos geradores, assim como prevista na legislação vigente, reduz o custo do descarte não reciclável e facilita o processo de reciclagem. A separação de resíduos pode ser incentivada pela planta de reciclagem, com diferentes práticas tarifárias e / ou políticas de conscientização pública.

Em contextos como ao do primeiro estudo de caso, o Poder Público também pode atuar como parceiro no fornecimento da área para a implantação da planta de reciclagem, dado o alto custo de aquisição do terreno. No segundo estudo, como a produção de agregado reciclado é destinada ao consumo interno, o desafio é garantir a quantidade de resíduos para suprir a quantidade necessária, uma vez que o custo de novos funcionários e equipamentos deve ser compensado pela diminuição dos custos de detonação presentes na produção de brita.

Nos dois casos, a redução do uso de agregados naturais (brita) e a redução de resíduos em aterros, a geração de empregos diretos e indiretos, juntamente com a compra e manutenção de equipamentos e construção, e o resultado financeiro positivo indicam ganhos ambientais, sociais e econômicos.

\section{AGRADECIMENTO}

Os autores agradecem ao CNPq e à CAPES pelo financiamento desta pesquisa e também às plantas de reciclagem e forncededores de equipamentos que contribuíram com as informações utilizadas no trabalho.

\section{REFERÊNCIAS}

ABRECON - Associação Brasileira para Reciclagem de Resíduos da Construção (2015) Pesquisa Setorial da Reciclagem de Resíduos da Construção 2014/2015. Relatório 2.

Agopyan V and John VM (2011) O Desafio da Sustentabilidade na Construção Civil. In: Goldenberg, J. (Ed.), Série Sustentabilidade, vol. 5. Blucher: São Paulo.

Ajayi SO, Oyedele LO, Bilal M, Akinade OO, Alaka HA, Owolabi AH and Kadiri KO (2014) Waste effectiveness of the construction industry: Understanding the impediments and requisites for improvement. Resources, Conservation \& Recycling 102: 101-112.

Al-Hajj A and Hamani K (2011) Material waste in the UAE Construction Industry: Main causes and minimization practices. Architectural Engineering and Design Management 7 (4): 221-235.

Bahera M, Bhattacharyya SK, Minocha AK and Maiti S (2014) Recycled aggregate from C\&D waste \& its use in concrete-A breakthrough towards sustainability in construction sector: $A$ review. Construction and Building Materials 68: 501-516.

Bakshan A, Srour I, Chehab G and El-Fadel M (2015) A field based methodology for estimating waste generation rates at various stages of construction projects. Resources, Conservation and Recycling 100: 70-80.

Brasil. Lei Federal 12.305/2010 (2010) Institui a Política Nacional de Resíduos Sólidos; Diário Oficial da União: Brasília, 2 de agosto. (Brazil. Federal Law 12,305 / 2010 (2010) Institutes the National Solid Waste Policy; Federal Official Gazette: Brasilia, August, 2)

Brasil. Lei Federal 10.522/2012 (2012) Institui o sistema de gestão sustentável de resíduos da construção civil e resíduos volumosos e o plano municipal de gerenciamento integrado de resíduos da construção civil e resíduos volumosos. Diário Oficial da União: Belo Horizonte, 24 ago.

Brasil. Resolução Conama n 307 (2002) Estabelece diretrizes, critérios e procedimentos para a gestão dos resíduos da construção civil. Diário Oficial da União: Brasília, 05 jul.

Cachim P, Velosa AL and Ferraz E (2014) Substitution materials sustainable concrete production Portugal. Journal of Civil Engineering 18(1): 60 -66.

Conseil International du Bâtiment, CIB (2011) Agenda XXI on Sustainable Construction.

Coelho A and Brito J (2011) Generation of construction and demolition waste in Portugal. Waste Management 
\& Research 29(7): 739-750.

Coelho A and Brito J (2012) Environmental analysis of a construction and demolition waste recycling plant in Portugal-Part II: environmental sensitivity analysis. Waste Management 33(1): 147-161.

Coelho A and Brito J (2013a) Environmental analysis of a construction and demolition waste recycling plant in Portugal-Part I: energy consumption and $\mathrm{CO} 2$ emissions. Waste management 33(5): 1258-1267.

Coelho A and Brito J (2013b) Economic viability analysis of a construction and demolition waste recycling plant in Portugal-part I: location, materials, technology and economic analysis. Journal of Cleaner Production 39: 338-352.

Consil of the European Union. Waste management and recycling: Concil adopts new rules (2018). Disponível em: https://www.consilium.europa.eu/pt/press/press-releases/2018/05/22/waste-management-and-recycling-council-adopts-new-rules/ (accesso em 10 de maio 2019).

Directive 2008/98/EC (2008). Waste Framework directive. Brussels, Belgium.

Huang WL, Lin DH, Chang NB and Lin KS (2002) Recycling of construction and demolition waste via a mechanical sorting process. Resources, Conservation and Recycling 37(1): 23-37.

Katz A and Baum H (2010). A novel methodology to estimate the evolution of construction waste in construction site. Waste Management 31(2): 353-358.

Kern A P, Dias MF, Kulakowski MP and Gomes LP (2015). Waste generated in high-rise buildings construction: $A$ quantification model based on statistical multiple regression. Waste Management 39:35-44.

Kern AP, Amor LV, Angulo SC and Montelongo AF (2018) Factors influencing temporary wood waste generation in high-rise buildings construction. Waste Management 78: 446-455.

Ledesma EF, Jiménez JR, Ayuso J, Fernández JM and Brito J (2015). Maximum feasible use of recycled sand from construction and demolition waste for eco-mortar production-Part-l: ceramic masonry waste. Journal of Cleaner Production 87: 692-706.

Martin J and Henrichs T (2015) The European environment state and outlook 2015 Chapter 4: natural resources and waste. Disponível em: https://www. eea.europa.eu/downloads/8d6424f22d6d0fabbac2d0177d9ce923/1464921025/chapter4.xhtml.pdf (accesso em 12 de dezembro de 2019)

Mália MAB, Brito J, Pinheiro MD and Bravo M (2013)
Construction and demolition waste indicators. Waste Management \&Research 31(3): 241-255.

Manowong E (2012) Investigating factors influencing construction waste management efforts in developing countries: an experience from Thailand. Waste Management 30(1): 56-71.

Melo AB, Gonçalves AF and Martins IM (2011) Construction and demolition waste generation and management in Lisbon (Portugal). Resources, Conservation and Recycling 55(12): 1252-1264.

Miranda LFR, Angulo SC and Carelli ED (2009) A reciclagem de resíduos de construção e demolição no Brasil: 1986-2008. Ambiente Construído 9 (1): 57-71.

Miranda LFR (2015) Inovações tecnológicas para a triagem de RCD. In: Seminário Nacional de Reciclagem de Resíduos da Construção Civil e Demolição. São Paulo, ABRECON.

Moyano PM and Agudo AR (2013) Selective classification and quantification model of C\&D waste from material resources consumed in residential building construction. Waste Management \& Research 31: 458-474. Nunes KRA, Mahlerb CF, Vallea R. and Neves C (2007) Evaluation of investments in recycling centers for construction and demolition wastes in Brazilian municipalities. Waste Management 27(11): 1531-1540.

Ortiz O, Pasqualino JC and Castells F (2010) Environmental performance of construction waste: comparing three scenarios from a case study in Catalonia, Spain. Waste Management 30(4): 646-654.

Pinto TP (1999) Metodologia para a gestão diferenciada de resíduos sólidos da construçãourbana. Tese (Doutorado)- Escola Politécnica da Universidade de São Paulo,São Paulo.

Ram V and Kalidindi SN (2017) Estimation of construction and demolition waste using waste generation rates in Chennai, India. Waste Management \& Research, 35(6): 610-617.

Sabai MM, Cox MGDM, Mato RR, Egmond ELC and Lichtenberg JJN (2013) Concrete block production from construction and demolition waste in Tanzania. Resources Conservation and Recycling 72: 9-19.

Sáez PV, Merino MDR, Porras-Amores C and González ASA (2014). Assessing the accumulation of construction waste generation during residential building construction works. Resources, Conservation and Recycling 93: 67-74.

Seadon JK (2010) Sustainable waste management systems. Journal of Cleaner Production 18(16): 1639-1651. Shima H, Tateyashiki H, Matsuhashi R and Yoshida Y 
(2005) An advanced concrete recycling technology and its applicability assessment through input-output analysis. Journal of Advanced Concrete Technology 3(1): 53-68.

Syymonds Group Ltd (1999) Construction and Demolition Waste Management Practices and Their Economic Impacts. Report to DGXI, European Commission, Final Report. Disponível em: https:// ec.europa.eu/environment/waste/studies/cdw_report.htm (accesso em 23 de outubro de 2019).

Tam VWY and Tam CM (2006) Evaluations of existing waste recycling methods: a Hong Kong study. Building and Environment 41(12): 1649-1660.

Tomasawa F, Noguchi T and Tamura M (2005) The way concrete recycling should be. Journal of Advanced Technology 3(1): 3-16.

Ulsen C (2011) Caracterização e separabilidade de agregados miúdos produzidos a partir de resíduos de construção e demolição. Tese (Doutorado) - Escola Politécnica, Universidade de São Paulo.

\section{AUTORES}

ORCID: https://orcid.org/0000-0001-6406-6250

ANDREA PARISI KERN, Dra. | UNISINOS - Universidade do Vale do Rio dos Sinos - PPGEC Programa de Pós Graduação em Engenharia Civil - São Leopoldo - Correspondência para: Av Unisinos, 950, CEP 92 022-000, RS | email: apkern@unisinos.br

ORCID: https://orcid.org/0000-0002-3896-1232

JOANA MICHELON MORESCO, M.Sc.| UNISINOS - Universidade do Vale do Rio dos Sinos - PPGEC Programa de Pós Graduação em Engenharia Civil - São Leopoldo - Correspondência para: Av Unisinos, 950, CEP 92 022-000, RS | email: joanamoresco@hotmail.com

ORCID: https://orcid.org/0000-0002-7575- 5649

EDUARDO PIZONI, M.Sc. | UNISINOS - Universidade do Vale do Rio dos Sinos - PPGEC Programa de Pós Graduação em Engenharia Civil - São Leopoldo - Correspondência para: Av Unisinos, 950, CEP 92 022-000, RS | email: pizoni.eduardo@gmail.com

ORCID: https://orcid.org/0000-0001-6406-6250

MARCO AURÉLIO STUMPF GONZÁLEZ, Dr. | UNISINOS Universidade do Vale do Rio dos Sinos - PPGEC Programa de Pós Graduação em Engenharia Civil - São Leopoldo Correspondência para: Av Unisinos, 950, CEP 92 022-000, RS | email: email: mgonzalez@unisinos.br

\section{COMO CITAR ESTE ARTIGO}

KERN, Andrea Parisi; MORESCO, Joana Michelon; PIZONI, Eduardo; GONZÁLEZ, Marco Aurélio Stumpf. Implantação De Plantas De Reciclagem De Rcd: Análise Financeira, Fatores De Influência E O Papel Dos Principais Agentes. MIX Sustentável, [S.I.], v. 7, n. 2, p. 79-92, abr. 2021. ISSN 24473073. Disponível em:<http://www.nexos.ufsc.br/index.php/mixsustentavel>. Acesso em: dia mês. ano. doi:https://doi. org/10.29183/2447-3073.MIX2021.v7.n2.79-92.

DATA DE ENVIO: 03/06/2020

DATA DE ACEITE: $12 / 11 / 2020$ 